\title{
Aprimorando a Qualidade de um Serviço Web através de Teste de Usabilidade - Uma Experiência Prática
}

\author{
Cristina Araújo ${ }^{1}$, Fábio $\operatorname{Santos}^{1}$, Leandro Galvão ${ }^{1}$, Tayana Conte ${ }^{1}$ \\ ${ }^{1}$ Departamento de Ciência da Computação \\ Universidade Federal do Amazonas (UFAM) - Manaus, AM - Brasil \\ \{crisoara, galvao, tayana\}@dcc.ufam.edu.br, faerickson@gmail.com
}

\begin{abstract}
Users' acceptability of Web applications relies strictly on these applications' usability, which can be improved through the use of evaluations. This paper reports a practical case study of usability testing, in which a synchronization service of navigation data across multiple devices, called Browserver is compared against a similar service. The results of this experiment show the possibility of conducting effective usability tests with low cost.

Resumo. A aceitabilidade das aplicações Web por seus usuários depende diretamente da usabilidade da aplicação, que pode ser melhorada através da realização de avaliações. Neste artigo, relatamos um caso prático de teste de usabilidade, no qual um serviço de sincronização de dados de navegação entre múltiplos dispositivos, chamado Browserver, é comparado com um serviço de finalidade semelhante. Os resultados desta experiência mostram a possibilidade de realizar testes de usabilidade eficazes com baixo custo.
\end{abstract}

\section{Introdução}

A demanda por desenvolvimento de aplicações Web vem crescendo sistematicamente nos últimos anos. Tal tendência pode ser observada não só do ponto de vista da quantidade de aplicações, mas também no que diz respeito à complexidade, gravidade e, conseqüentemente, aos riscos para o negócio de diversas organizações fortemente apoiadas por aplicações Web. Adicionalmente, a exploração de conceitos de ubiqüidade e facilidade de acesso a informações e serviços proporcionados pela Web vem permitindo o uso cada vez mais intenso dessas aplicações na sociedade (KAPPEL et al. 2006), apoiando as relações de negócio entre empresas e indivíduos em atividades do dia-a-dia relacionadas ao trabalho ou lazer.

A fim de oferecer um serviço Web de alta qualidade, decidiu-se realizar avaliações específicas em critérios de qualidade relevantes para esse tipo de aplicação. Segundo OFFUTT (2002), a usabilidade é um dos três critérios de qualidade que deve dirigir o processo de desenvolvimento em organizações que produzem aplicações Web.

A norma ISO 9241-11 (ISO 1998) define usabilidade como "a medida pela qual um produto pode ser usado por usuários especificos para alcançar objetivos específicos com eficácia, eficiência e satisfação em um contexto específico de uso". No caso de aplicações $W e b$, a importância da usabilidade é ainda maior se comparada à maioria das aplicações de software tradicionais, devido às suas próprias características, pois "são aplicações interativas, centradas no usuário e baseadas em hipermídia, onde a interface com o usuário desempenha um papel central" (OLSINA et al. 2006). 
Este artigo apresenta e relata uma experiência prática de teste de usabilidade que foi conduzido com o objetivo de caracterizar a serviço Browserver. Este permite que usuários do navegador Firefox tenham acesso ubíquo a dados de navegação Web (p.ex. favoritos, histórico, dados de formulário, etc.), mediante login/senha. Os parâmetros tempo de execução das tarefas e número de erros cometidos pelo usuário ao realizá-las foram comparados aos de outro serviço Web com finalidade semelhante.

O objetivo deste artigo é descrever como esse teste foi planejado e executado e analisar seu custo-eficiência, de maneira a fornecer informações que possam incentivar outras empresas de desenvolvimento a realizar avaliações de usabilidade. Com isso, espera-se contribuir para a melhoria da qualidade de produtos de software em um dos mais importantes critérios de aceitação de aplicações interativas em geral, e em particular para aplicações Web (HITZ et al. 2006).

O restante do artigo está organizado da seguinte forma: a Seção 2 apresenta um breve referencial teórico sobre avaliações de usabilidade. A Seção 3 descreve resumidamente o serviço Web Browserver. A Seção 4 descreve o planejamento e a execução do teste de usabilidade, detalhando suas etapas e papéis envolvidos. A Seção 5 discute os resultados e aprimoramentos do serviço Web a partir do teste de usabilidade. Por fim, a Seção 6 apresenta as conclusões e lições aprendidas com essa experiência prática.

\section{Avaliações de Usabilidade}

Ao avaliar sistemas com relação à qualidade de uso, deve-se considerar que, do ponto de vista do usuário, a qualidade da interface e da interação determina a qualidade do sistema, e não seus algoritmos, arquitetura ou modelos de dados (PRATES e BARBOSA (2003). Portanto, para o usuário, o sistema é a interface. Problemas de usabilidade tornam uma aplicação ineficaz, ineficiente ou difícil de ser utilizada para um usuário (MATERA et al. 2006). Esses problemas podem ser detectados através de métodos de avaliação diversos, realizados ao longo do processo de desenvolvimento.

Os métodos comumente adotados para avaliação de usabilidade podem ser divididos em duas grandes categorias: (1) Inspeções de Usabilidade (ou Métodos Analíticos (PRATES e BARBOSA 2003)), nas quais inspetores (normalmente especialistas) examinam aspectos relacionados à usabilidade da aplicação, para detectar violações de princípios de usabilidade estabelecidos; e (2) Testes de Usabilidade, que se baseiam na participação direta de usuários, os quais executam tarefas pré-definidas e comentam sobre a interface, enquanto que observadores registram as dificuldades encontradas e o sucesso ou insucesso na realização das tarefas.

O teste de usabilidade é considerado o método mais eficaz em avaliar sistemas e protótipos do ponto de vista do usuário das aplicações. Porém freqüentemente seus custos são altos, pois envolvem o tempo dos usuários e muitas vezes o uso de laboratórios específicos de usabilidade (MATERA et al. 2002). Além disso, os testes de usabilidade também apresentam alguns inconvenientes, tais como a dificuldade de selecionar uma amostra representativa da população (MATERA et al. 2006).

NIELSEN (1994) argumenta que uma razão importante para a engenharia de usabilidade não ser comumente utilizada em projetos de desenvolvimento é o custo presumido de utilização das suas técnicas. No entanto, é possível realizar avaliações de usabilidade de baixo custo, como por exemplo, realização de testes de usabilidade simplificados, com uso do método Thinking-aloud e de 3 a 5 participantes, o que 
maximiza a relação custo-benefício do teste (NIELSEN 2000). Isto porque o teste com um usuário é capaz de identificar aproximadamente 30\% dos problemas da aplicação. Cada novo usuário encontra $30 \%$ de problemas, destes uma parte representa novos problemas, enquanto a outra representa problemas encontrados pelos usuários anteriores. Então a cada novo teste se reduz o número de novos problemas e se aumenta o número de problemas já encontrados. Segundo NIELSEN (2000), com cinco usuários, é possível encontrar aproximadamente $85 \%$ dos problemas da aplicação e o benefício dos novos erros encontrados vale o custo do teste executado.

Através de testes de usabilidade, podemos observar o comportamento dos usuários que realizam a mesma tarefa. $\mathrm{O}$ uso do método Thinking-Aloud e o registro das verbalizações durante o teste permitem que sejam compreendidas as dificuldades sentidas pelo usuário. Maneiras novas (muitas vezes inesperadas e não desejadas) do usuário operar o sistema aparecem. Isso permite descobrir erros e consertá-los ainda no início do projeto, quando são mais baratos e fáceis de ajustar.

\section{Caracterização do Serviço Web - Browserver}

O Browserver (= browser + server) é um serviço Web que possibilita o armazenamento e recuperação de dados de navegação Web, tais como dados de cookies, abas abertas, dados de formulários, histórico e lista de extensões instaladas no navegador Firefox.

A versão do Browserver testada foi a 0.3. Uma extensão (addon) deve ser instalada no navegador para coletar e recuperar os dados de navegação. No lado servidor, um repositório armazena tais dados mediante login/senha.

A Figura 1 ilustra o cenário básico de uso do serviço de Browserver. Uma pessoa acessa páginas Web utilizando um dispositivo qualquer (de origem). Enquanto isso, o Browserver Addon sincroniza em background os dados que ela vai produzindo (passos 1 e 2). Mais tarde, essa pessoa passa a utilizar outro dispositivo (de destino) para continuar a navegar na Web (passo 3). Nesse momento, o usuário se conecta de forma segura ao serviço de Browserver, que sincroniza os dados de navegação (passo 4), promovendo uma verdadeira continuidade da experiência de navegação.

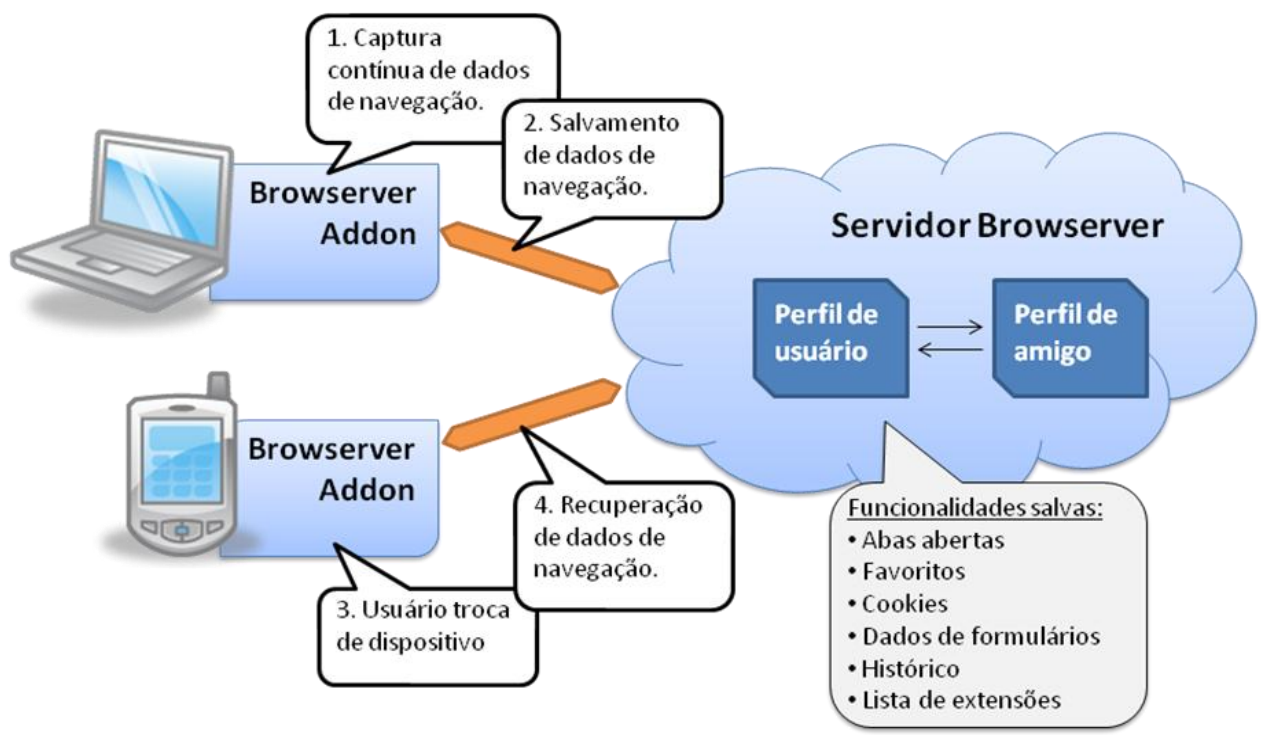

Figura 1 - Arquitetura do Serviço Browserver 


\title{
4. Avaliação de Usabilidade
}

Como este era o primeiro teste de usabilidade do Browserver, optou-se por realizar uma comparação entre o Browserver e outro serviço Web de mesmo propósito. Foi feito um estudo para analisar quais outros serviços Web oferecem recursos semelhantes ao Browserver e foi escolhido um serviço como base de comparação. Esta comparação entre serviços semelhantes é detalhada em (CARVALHO et al. 2010) . O serviço Web escolhido apresentava os mesmos serviços oferecidos pelo Browserver dentre eles: 1Adição e exclusão de abas, 2 - Sincronização de abas e 3 - Salvamento de cookies e 4 Auto preenchimento de formulários.

De acordo com o paradigma GQM (BASILI e ROMBACH 1988), o objetivo do teste de usabilidade é analisar a usabilidade do Browserver com o propósito de caracterizá-lo em relação ao tempo de realização de atividades e percentual de acertos, em comparação com um serviço Web semelhante, do ponto de vista dos pesquisadores, no contexto de um teste de usabilidade efetuado com usuários especialistas.

Um bom teste de usabilidade deve incluir as atividades estratégicas para o bom funcionamento do software. No caso do Browserver, estas atividades foram:

1- Download e instalação de add-on do serviço Web;

2- Criação de Conta de Usuário no serviço Web;

3- Abertura de abas, utilização de cookies e autopreenchimento de formulários;

4- Sincronização entre o computador e o servidor dos dados de navegação Web.

5- Sincronização entre o servidor e o computador para recepção dos dados de navegação anteriormente gravados.

\begin{abstract}
Baseado nas recomendações propostas por (PRATES e BARBOSA 2003), o processo de teste de usabilidade foi dividido em três etapas: planejamento, execução e análise, as quais são descritas na Tabela 1. Os perfis necessários para a execução dos testes são chamados papéis (PREECE et al. 2005), que são apresentados na
\end{abstract}

Tabela 2.

Tabela 1 - Atividades e Etapas do Processo de Teste de Usabilidade

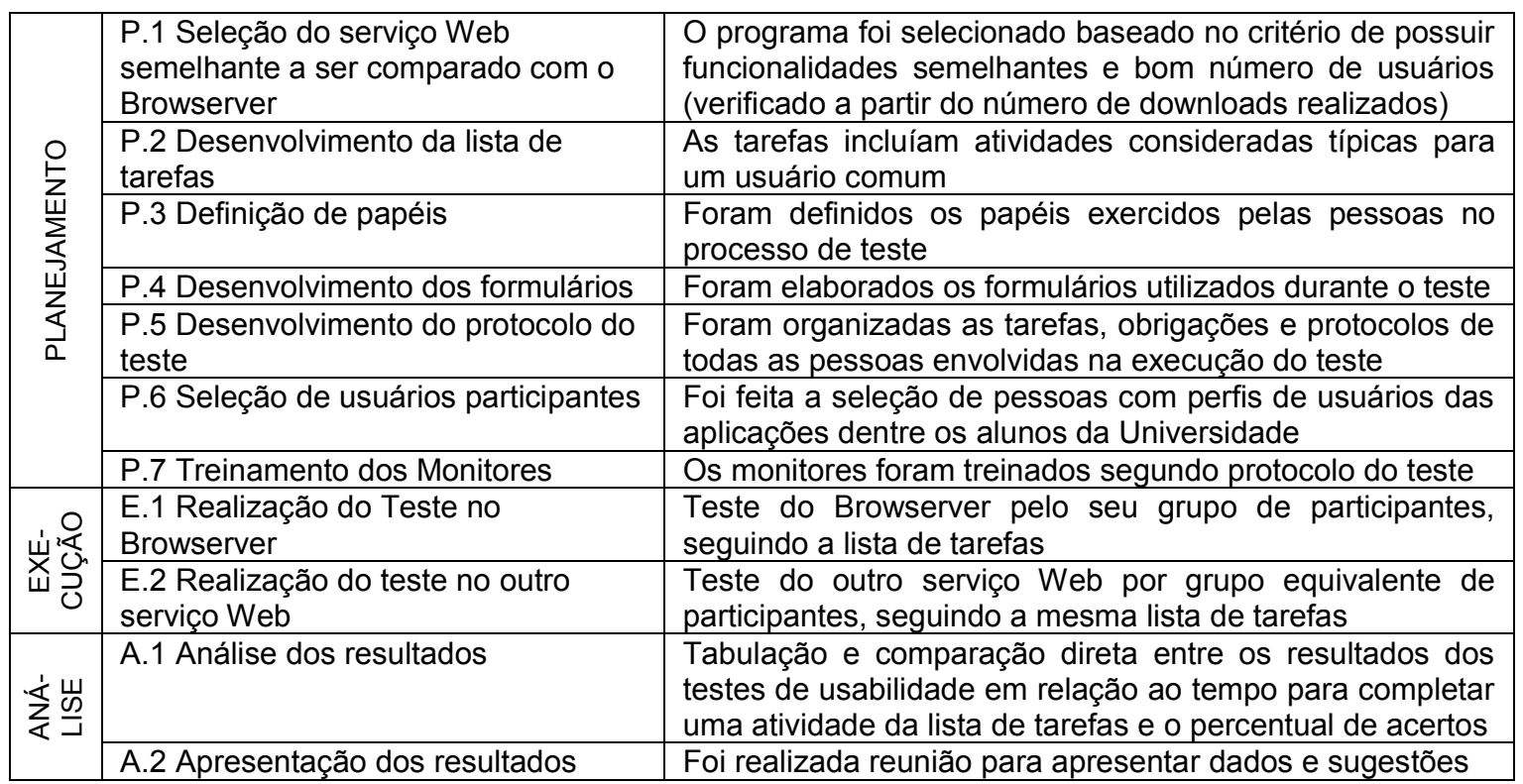


Tabela 2 - Papéis envolvidos no Teste de Usabilidade

\begin{tabular}{|l|l|}
\hline Criadores do Browserver & Especialistas em redes e serviços Web que desenvolveram o Browserver \\
\hline Uspecialistas em & $\begin{array}{l}\text { Especialistas em avaliação de usabilidade que foram contatados pelos } \\
\text { Criadores do Browserver para realizar o teste de usabilidade }\end{array}$ \\
\hline Moderador & $\begin{array}{l}\text { Responsável pela execução do processo de teste. Esse papel foi exercido } \\
\text { por um dos especialistas em usabilidade. Outro especialista atuou como } \\
\text { apoio e controle de qualidade durante o processo de teste }\end{array}$ \\
\hline Monitor & $\begin{array}{l}\text { Pessoa com bom conhecimento de usabilidade. Juntamente com o } \\
\text { moderador, acompanhou cada teste individualmente, anotando os } \\
\text { resultados observados de cada tarefa realizada pelo Participante }\end{array}$ \\
\hline Participantes & $\begin{array}{l}\text { Responsáveis pela execução da lista de tarefas. Os quatorze participantes } \\
\text { assinaram um Termo de Consentimento Livre e Esclarecido e preencheram } \\
\text { um formulário de caracterização. Foram classificados em dois grupos } \\
\text { homogêneos, de acordo com os critérios de experiência com o uso de } \\
\text { plugins/add-ons e de tempo de acesso à internet: sete deles testaram o } \\
\text { Browserver e ou outros sete testaram o serviço comparado }\end{array}$ \\
\hline
\end{tabular}

\section{Execução do Teste de Usabilidade}

\subsection{Ambiente de Teste}

Para a realização dos testes, foi utilizado um ambiente de baixo custo, consistido de apenas duas estações de trabalho com Ubuntu 9.04, navegador Firefox 3.5 e acesso à Internet de $2 \mathrm{Mbps}$. Como as tarefas simulavam a troca de máquinas, foram criados dois usuários no sistema operacional. Os dados pessoais de navegação são vinculados à pasta / home do usuário. Assim, trocando de usuário, houve a simulação do uso de uma máquina diferente. O participante tinha na lista de tarefas a marcação de quando deveria trocar de usuário.

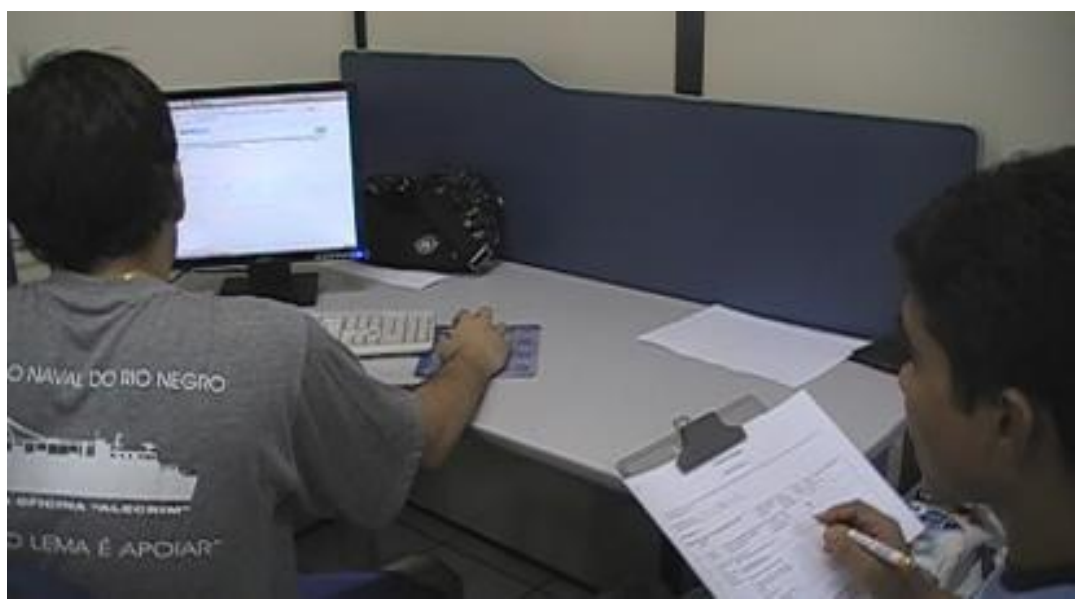

Figura 2 - Realização de teste pelo participante observado pelo moderador

\subsection{Realização dos Testes}

Ao começar a sessão dos testes, o monitor/moderador leu para o participante o Roteiro de Orientação do Teste. Em seguida, o monitor apresentou a Lista de Tarefas ao participante, o qual foi solicitado para ler em "voz alta" as tarefas e expressar dúvidas e dificuldades também em "voz alta" (método Thinking Aloud).

Após verificar se o participante entendeu os procedimentos, o monitor começou a conduzir os testes, pedindo ao participante que executasse cada tarefa e o avisasse ao concluir. Enquanto isso, o monitor cronometrava o tempo, anotando os comentários, 
dúvidas e erros do participante no formulário de Coleta de Dados. Ao término dos testes, cuja duração levou cerca de uma hora, o monitor entregou a cada participante um formulário de feedback, para expressar as impressões sobre serviço Web testado.

\section{Resultados do Teste e Aprimoramentos no Browserver}

Conforme descrito na Seção 4, para caracterizar a usabilidade do Browserver neste teste, mediu-se o tempo que os usuários levaram para completar as tarefas e o seu percentual de acertos. A Tabela 3 apresenta o resultado do teste em termos de Tempo para Execução e Percentual de Acertos do Browserver e do serviço Web comparado.

Tabela 3 - Resultado do Teste em Tempo e Percentual de Acertos

\begin{tabular}{|c|c|c|c|c|c|c|}
\hline & \multicolumn{3}{|c|}{ Tempo em segundos } & \multicolumn{3}{|c|}{ Acertos (\%) } \\
\hline Tarefas & $\begin{array}{c}\text { Com- } \\
\text { parado }\end{array}$ & $\begin{array}{l}\text { Brow- } \\
\text { server }\end{array}$ & $\begin{array}{l}\text { Dife- } \\
\text { rença }\end{array}$ & $\begin{array}{c}\text { Com- } \\
\text { parado }\end{array}$ & $\begin{array}{l}\text { Brow- } \\
\text { server }\end{array}$ & $\begin{array}{l}\text { Dife- } \\
\text { rença }\end{array}$ \\
\hline $1.1-$ Abrir Firefox & 24 & 19 & 5 & 100 & 100 & 0 \\
\hline 1.2 - Acessar o site do serviço & 144 & 81 & 63 & 100 & 100 & 0 \\
\hline 1.3 - Instalar o serviço & 108 & 95 & 13 & 100 & 100 & 0 \\
\hline 1.4 - Criar Conta de usuário & 338 & 374 & -36 & 100 & 42 & 58 \\
\hline $1.5-$ Sincronizar & 119 & 115 & 4 & 57 & 28 & 29 \\
\hline 1.6 - Abrir uma aba e acessar site de notícias & 45 & 12 & 33 & 100 & 85 & 15 \\
\hline 1.7 - Sem fechar a aba anterior, abrir o blog $X$ & 240 & 168 & 72 & 100 & 85 & 15 \\
\hline 1.8 - Sem fechar a aba anterior, abrir o site $Y$ & 87 & 69 & 18 & 100 & 85 & 15 \\
\hline $1.9-$ Sincronizar & 81 & 30 & 51 & 57 & 57 & 0 \\
\hline 1.10 - Fechar Firefox & 11 & 10 & 1 & 100 & 85 & 15 \\
\hline $2.1-$ Abrir Firefox & 17 & 17 & 0 & 100 & 100 & 0 \\
\hline 2.2 - Acessar o site do serviço & 89 & 85 & 4 & 100 & 100 & 0 \\
\hline 2.3 - Instalar o serviço & 41 & 107 & -66 & 100 & 100 & 0 \\
\hline $2.4-$ Fazer login & 110 & 72 & 38 & 100 & 57 & 43 \\
\hline 2.5 - Sincronizar & 241 & 151 & 90 & 57 & 42 & 15 \\
\hline 2.6 - Verificar se as abas foram abertas & 80 & 166 & -86 & 0 & 42 & -42 \\
\hline 2.7 - Sem fechar a aba anterior, abrir outra aba & 29 & 31 & -2 & 100 & 100 & 0 \\
\hline 2.8 - Fechar a aba do site de notícias & 13 & 13 & 0 & 14 & 28 & -14 \\
\hline $2.9-$ Sincronizar & 52 & 13 & 39 & 100 & 42 & 58 \\
\hline 2.10 - Fechar Firefox & 7 & 12 & -5 & 100 & 100 & 0 \\
\hline $3.1-$ Abrir Firefox & 27 & 18 & 9 & 100 & 100 & 0 \\
\hline $3.2-$ Fazer Login & 66 & 39 & 27 & 71 & 71 & 0 \\
\hline $3.3-$ Sincronizar & 189 & 21 & 168 & 85 & 42 & 43 \\
\hline $3.4-$ Verificar se as abas foram a & 48 & 26 & 22 & 0 & 42 & -42 \\
\hline 3.5 - Fechar Firefox & 13 & 15 & -2 & 100 & 100 & 0 \\
\hline
\end{tabular}

\subsection{Aprimoramentos do Browserver com relação ao Tempo}

Conforme dados da Tabela 3, os participantes completaram as tarefas no Browserver mais rapidamente, devido à menor quantidade de passos que tinham para realizar as tarefas. Contudo, muitos participantes ficaram com dúvidas se já haviam concluído a tarefa no Browserver, pois não foram exibidas mensagens de feedback. Portanto deramnas erroneamente como concluídas.

As tarefas que os participantes dos testes do Browserver tiveram mais dificuldades e demoraram mais tempo para concluí-las foram Criação de conta (1.4), Instalação (2.3) e Verificação da abertura de abas (2.6).

\subsection{Aprimoramentos do Browserver com relação aos Acertos}

Os participantes do Browserver tiveram menos acertos na realização das tarefas em relação ao serviço comparado. Enquanto que os participantes que avaliaram o serviço 
comparado completaram com sucesso 17 das 25 tarefas $(68 \%)$, os participantes do Browserver completaram com sucesso apenas 10 tarefas (40\%).

Os principais erros do serviço comparado foram causados pela mudança de paradigma na questão de salvar abas. Enquanto o Browserver reabre no destino as abas abertas na origem, o serviço comparado mantém uma lista das abas, acessível apenas pelo menu do navegador, o que não é intuitivo ao usuário iniciante.

Os principais erros do Browserver foram nas tarefas de Criação de conta (1.4), Verificação da abertura de abas (2.6) e todas as tarefas de Sincronização (1.9, 2.5, 2.9 e 3.3 ), as quais são tarefas interdependentes, pois se o participante não conseguia criar a conta, não conseguia salvar as abas e nem, portanto, verificar se elas eram abertas nos dispositivo de destino.

\subsection{Sugestões Apresentadas}

Após a análise da tabulação dos dados e a reunião com a equipe de desenvolvimento, foram sugeridas as seguintes melhorias quanto às seguintes tarefas:

Sincronização. Embora os desenvolvedores diferenciem se os dados de navegação são sincronizados do computador para o servidor ou vice-versa, o usuário deseja apenas que os dados locais reflitam o que está gravado no servidor. Portanto, os menus "Salvar" e "Carregar" devem ser mesclados em apenas um (Sincronizar).

Cadastro de Usuário. Os menus e indicadores dessa tarefa devem ser reposicionados ou renomeados. Assim eles serão mais facilmente vistos e encontrados pelos usuários.

Feedback. O Browserver deve avisar a conclusão de todas as ações iniciadas pelo usuário, seja por meio de mudança de estado na barra de status do navegador, ou meio de mensagens pop-up.

\section{Lições Aprendidas e Considerações Finais}

Este trabalho apresentou o relato da execução de um teste de usabilidade de um serviço Web. Pode-se observar que é viável realizar um teste de usabilidade com poucos recursos, pois, no caso de uso estudado, foram utilizados apenas recursos locais (dois PCs comuns), o que implicou em um baixo custo geral do teste de usabilidade, pois não houve custo adicional em relação a hardware e software. Os próprios participantes do teste foram voluntários, o maior custo foi relacionado ao tempo dos especialistas, pois se levou 24 horas para o planejamento, 16 horas na execução dos testes e 8 horas na análise. Espera-se com estes resultados incentivar a indústria de software a realizar avaliações de usabilidade com maior freqüência.

Uma lição aprendida foi sobre a importância de realizar testes de usabilidade a partir do primeiro módulo ou do protótipo de interação definido. Quanto mais cedo se avalia a usabilidade, maior é o ganho, caso o sistema siga um padrão de interação. Nesta experiência, que envolveu a avaliação de um serviço Web, observou-se que a versão mudava muito rapidamente. Daí a necessidade de fazer documentos padrões para que não tivéssemos que mudá-los a cada nova versão, apenas mudávamos o documento de Coleta de Dados que era onde estavam o passo a passo necessário para executar a tarefa.

Um ganho observado ao realizar o teste empregando Thinking Aloud, foi que o ato de "pensar em voz alta" também incentivou o participante a discutir as dificuldades na realização das tarefas com o monitor, o que facilitou a observação do teste. 
Como continuação do presente trabalho, será feito um mockup (protótipo em wireframe) com as sugestões de melhorias que foram apresentadas à equipe de desenvolvimento. A elaboração da nova versão do software será feita de acordo com esse protótipo. Também será realizado um novo teste de usabilidade com a nova versão após melhorias. Pretende-se fazer uma comparação qualitativa dos resultados dos dois testes.

Como questão de pesquisa para futuros estudos, gostaríamos de avaliar quantitativamente o ganho para projetos ao se realizar avaliações prévias de usabilidade através do uso de mockups durante etapa de análise/ projeto do software.

\section{Agradecimentos}

Os autores agradecem a todos os participantes do teste de usabilidade, aos desenvolvedores do Browserver e ao Prof. Edjard Mota. Agradecemos ao CNPq e à FAPEAM pelo apoio.

\section{Referências}

BASILI, V., ROMBACH, H., 1988. "The tame project: towards improvement-oriented software environments." IEEE Transactions on Software Engineering, v. 14, n. 6, pp. 758 - 773.

CARVAlHO, L. G., VAlle, R., PASSITO, A., MOTA, E., MOTA, E., NOVELlinO, R., PENARANDA, A., 2010. Synchronizing Web Browsing Data with Browserver. Submetido ao IEEE Symposium on Computers and Communications (ISCC).

HITZ, M., LEITNER, G., MELCHER, R., 2006. "Usability of Web Applications".In: Kappel, G., Pröll, B., Reich, S., Retschitzegger, W. (eds), Web Engineering: The Discipline of Systematic Development of Web Applications, Chapter 11, John Wiley $\backslash \&$ Sons.

ISO (1998). ISO 9241-11: Ergonomic requirements for office work with visual display terminals (VDTs) - Part 11: Guidance on usability, International Organization for Standardization.

KAPPEL, G., PRÖLL, B., REICH, S., RETSCHITZEGGER, W., 2006. "An Introduction to Web Engineering".In: Kappel, G., Pröll, B., Reich, S., Retschitzegger, W. (eds), Web Engineering: The Discipline of Systematic Development of Web Applications, John Wiley \& Sons.

MATERA, M., COSTABILE, M. F., GARZOTTO, F., PAOLINI, P., 2002. "SUE Inspection: An Effective Method for Systematic Usability Evaluation of Hypermedia." IEEE Transactions on Systems, Man and Cybernetics, Part A, v. 32, n. 1, pp. 93-103.

MATERA, M., RIZZO, F., CARUGHI, G. T., 2006. "Web Usability: Principles and Evaluation Methods".In: Mendes, E., Mosley, N. (eds), Web Engineering, Chapter 5, New York, Spinger Verlag.

NIELSEN, J., 1994. "Guerrilla HCI: using discount usability engineering to penetrate the intimidation barrier"(eds), Cost-justifying usability, Orlando, USA, Academic Press.

NIELSEN, J. (2000). "Why You Only Need to Test with 5 Users." Jakob Nielsen's Alertbox, Disponível em http://www.useit.com/alertbox/20000319.html Acessado em 31/01/2010.

OFFUTT, J., 2002. "Quality Attributes of Web Software Applications." IEEE Software, v. 19, n. 2, pp. 25-32.

OLSINA, L., COVELLA, G., ROSSI, G., 2006. "Web Quality".In: Mendes, E., Mosley, N. (eds), Web Engineering, Chapter 4, Spinger Verlag.

PRATES, R. O., BARBOSA, S. D. J., 2003. "Avaliação de Interfaces de Usuário - Conceitos e Métodos".In: Coello, J. M. A., Fabbri, S. C. P. F. (eds), Jornada de Atualização em Informática do Congresso da Soc. Brasileira de Computação, Cap. 6, Campinas, SBC.

PREECE, J., ROGERS, Y., SHARP, H., 2005. Design de Interação: Além da Interação Homem-Computador. Porto Alegre, Bookman. 